\title{
On Finiteness in the Card Game of War
}

\author{
Evgeny Lakshtanov and Vera Roshchina
}

\begin{abstract}
The game of war is one of the most popular international children's card games. In the beginning of the game, the pack is split into two parts, then on each move the players reveal their top cards. The player having the highest card collects both and returns them to the bottom of his hand. The player left with no cards loses. It is often wrongly assumed that this game is deterministic and the result is set once the cards have been dealt.However, it is not quite so; as the rules of the game do not prescribe the order in which the winning player will put his take to the bottom of his hand: own card, then rival's or vice versa: rival's card, then own. We provide an example of a cycling game with fixed rules. Assume now that each player can seldom but regularly change the returning order. We have managed to prove that in this case the mathematical expectation of the length of the game is finite. In principle it is equivalent to the graph of the game, which has got edges corresponding to all acceptable transitions, having got the following property: from each initial configuration there is at least one path to the end of the game.
\end{abstract}

\section{The Game of War}

The Game of War is an internationally popular children's card game with a very basic set of rules. At the start, the pack is shuffled and divided into two equal parts; then each player reveals their top card. The player with the highest value card then collects both cards and returns them to the bottom of their hand. This process continues until one player loses all cards.

Many who play this game in childhood lose patience before the end as the back and forth nature of card transfer often allows a player to almost reach the point of victory only to see their hand reduced dramatically in a matter of moments. In effect children playing are conducting basic mathematical experiments and observing the development of a dynamic system. It is often wrongly assumed that this game is deterministic and the result is set once the cards have been dealt. However this is not so; the rules of the game do not stipulate in which order the winner of each play returns the cards to the bottom of their hand - own card first and then rival's or vice versa.

We shall at first consider a model with an arbitrary (even) number of cards and only one suit. Hence, a situation where both players present the same value card cannot occur, in the classic game, if this does occur, it would lead to a 
process called 'war' and players would continue to lay cards until one plays a winner, thus claiming all cards played.

So consider a game where the players strictly control the order in which the cards are returned to the bottom of their hand; in this case there is a chance that the game will never finish. Such a cyclic game is shown in Fig. 1 where $n=6$. The card of the 'left' player is always returned to the bottom of the winner's hand before the card of the 'right' player. Such a never ending game

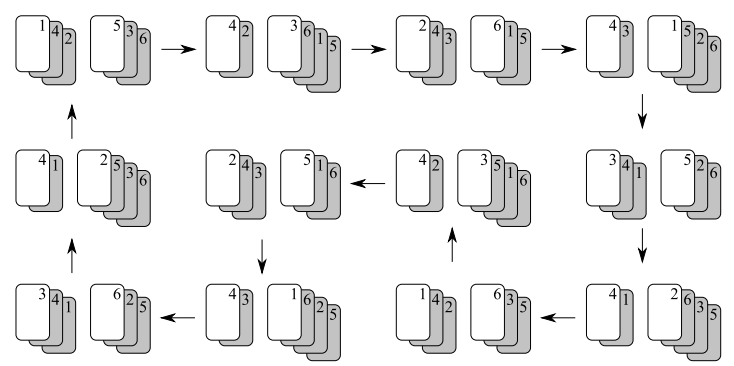

Figure 1: A never ending game, $n=6$.

can also occur with a standard deck if the above rules are followed. An example of an initial shuffling for such a game is given on Fig. 2 (throughout the paper we assume that the ace is the highest value card). Observe that after the first two moves are made, the last two cards in the hand of player $L$ are $(A \oslash, K \mathbf{p})$, and in the hand of player $R-(K \odot, A \boldsymbol{p})$, then after the next two moves the last 4 cards are $(A \odot, K \boldsymbol{q}, A \diamond, K \mathbf{p})$ for the player $L$ and $(K \odot, A \boldsymbol{q}, K \diamond, A \boldsymbol{\mathbf { p }})$ for player $R$, and so on. It can be easily seen that the order of the card values is preserved, and after 26 moves the players will end up with precisely the same distribution of card values in their hands (although suits would get shuffled). Note that in this particular case 'war' never happens, because the cards played on each move always have different values.

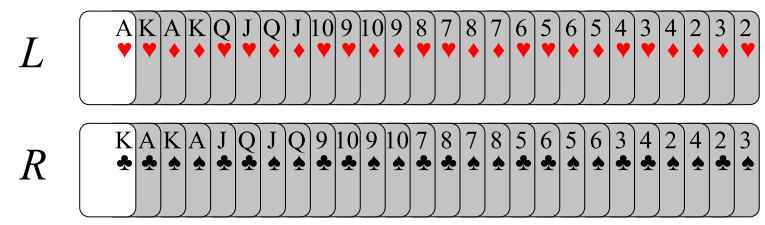

Figure 2: A never ending game, standard pack of 52 cards.

Hence we have established that when rigid rules are used, it might be possible to never finish the game. But what if players use both possible ways of returning cards to their hands, and on each particular move choose such rules at random? In this case, it is not immediately clear whether the players have a nonzero chance to reach the end of the game, however, it is possible to give an answer to this question using a probabilistic model of the game. We show that if the players use both rules to return the cards to their hand, the mathematical 
expectation of the number of moves in the game is finite, (i.e., there is a zero chance of never finishing the game).

\section{Mathematical model}

We now focus on a model case in which there are cards from 1 to $n$. We assume that each player possesses certain peculiarities which means when collecting cards their card will be placed on the bottom with probability $p_{1}^{i}>0$ and second from bottom with $p_{2}^{i}>0$, where the index $i$ identifies the player $(i$ is either $L$ or $R$ ). This is illustrated on Fig. 3 where player $L$ wins, and chooses to place his card on the bottom with probability $p_{1}^{L}$, and uses the other rule with probability $p_{2}^{L}$. Let $C$ be the deck of cards, either numbered (from 1 to

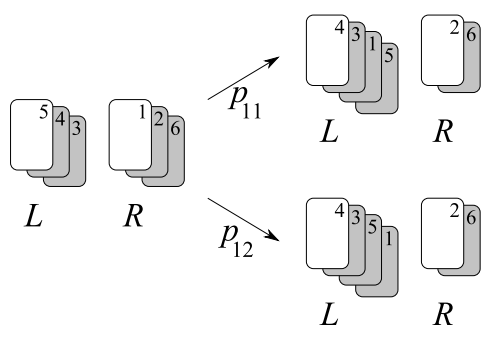

Figure 3: Probabilities with which player $L$ chooses the order to place the cards in his hand (model pack of 6 cards)

$n$, where $n$ is any even number) or standard (with 52 cards, valued from 2 to ace and having 4 suits). We use $L$ to denote the cards in the hand of the 'left' player, and $R$ to denote the 'right' player's hand. In the model case, $L$ and $R$ are ordered sets of numbers, one of which can be empty; this corresponds to the end of the game.

Each division of the deck into two ordered sets is called a game state. That is, the game starts with a state with both sets $L$ and $R$ having an equal number of members, and ends with the state in which one of the sets is empty (the final state). Each game play (when the players show their top cards, compare them and then put the ordered pair to the bottom of the winner's hand) is a transition from one state to another, because it starts with two ordered sets of cards, and ends with two (different) ordered sets, which correspond to the players' hands.

Such dynamics in which a transition to the next state happens with fixed probabilities independent on the preceding choices is called Markov chain. In the theory of Markov chains, one of the fundamental facts is the following. Assume that any initial state is possible. The mathematical expectation of the number of moves before the absorption (reaching the final state) is finite if and only if the final state can be reached from any state [3, Chap.3]1 1 Usually a Markov chain

\footnotetext{
${ }^{1}$ Let us explain this fact. Assume that termination is possible from every initial state. It means that for every vertex $v_{i}$ there exists $N_{i}$ such that the conditional probability $P\left(\cdot \mid v_{i}\right)$
} 
is represented as a directed graph in which the vertices correspond to states, and edges correspond to the transitions. An edge leaves one vertex and reaches another if and only if there exists a transition from the former to the latter with a nonzero probability. It is not difficult to see that in our case each non-final vertex (or state) has only two outgoing edges: once both players have revealed their cards, the winner, by putting the top cards to the bottom of his hand, defines such two transitions with probabilities $p_{1}^{i}$ and $p_{2}^{i}$, where $i$ is either $L$ or $R$, depending on who wins this particular game. We call a vertex attaining if it has a final state as one of its descendants and wandering otherwise. It is obvious that a descendant of a wandering vertex is again wandering, and a predecessor of an attaining one is again attaining. A graph is called absorbing if all the vertices are attaining. That is, the graph of our game is absorbing if and only if for every state (each division on the pack into two hands) it is possible to finish playing the game in a finite number of moves. The difference between absorbing and non-absorbing graphs can be seen in Fig. 4. Both graphs i) and ii) have

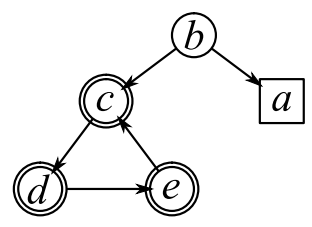

i)

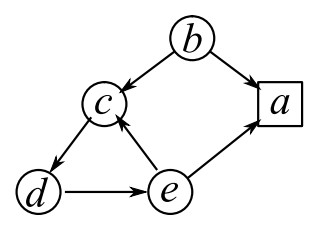

ii)

Figure 4: Difference between absorbing and non-absorbing graphs: the graph $i$ ) is not absorbing, but the graph ii) obtained by adding one extra edge between $e$ and $a$ to the graph $i$ ) is absorbing.

the same final state $a$. Observe that the graph $i$ ) is not absorbing. It can be easily seen that it is impossible to reach the state $a$ from any of the vertices $c$, $d$ and $e$, while the graph ii), which differs from $i$ ) only by one additional edge that leads from $e$ to $a$, is absorbing, as one can get into $a$ from any other vertex.

\section{Proof of the main result}

We have already established that each state (except for final) has exactly two outgoing edges. Now assume that each of the players has at least two cards in their hands. There are exactly two incoming edges corresponding to the possible preceding plays in which either of the players won (see Fig. 5 for an illustration for a game with 6 cards).

to terminate the game during next $N_{i}$ steps is positive. Since our graph is finite, taking $N=\max \left\{N_{i}\right\}$ and denoting $q>0$ the minimum over i of such probabilities, we see that the unconditional probability to terminate the game in at most $N$ steps is at least $q>0$. So the probability to stay in the game $k N$ steps is at most $(1-q)^{k}$. From this, the probability to stay in the game $n$ steps converges to zero faster than a geometric progression. 


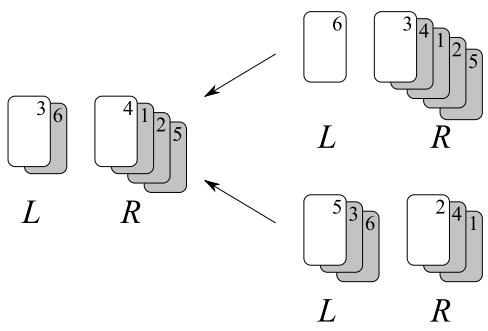

Figure 5: If the players have at least two cards each, then this vertex has exactly two predecessors.

If one of the players has only one card left, he could not have won the preceding play, and hence there is only one possibility for the winner. This is illustrated on Fig. 6 for a game with 6 cards.

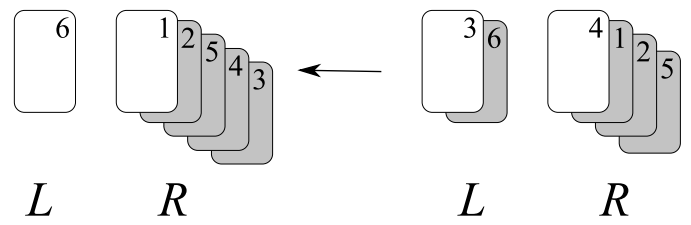

Figure 6: If one of the players has only one card left, this vertex has a unique direct predecessor.

Using this crucial observation, we are going to show that the game graph has no wandering vertices (i.e. it is possible to finish the game starting from any state). We show this by assuming that there is at least one wandering vertex, and then establishing that this leads to a contradiction.

By $\mathcal{W}$ denote the set of all wandering vertices in our graph. Let $m$ be the number of vertices in $\mathcal{W}$. Every vertice in $\mathcal{W}$ has exactly 2 descendants, so the number of edges leaving from $\mathcal{W}$ is $2 m$. Observe that each edge going out from a wandering vertex goes into a wandering vertex again, otherwise we would get a contradiction with the definition of a wandering vertex.

Therefore, the total number of different edges that lead into $\mathcal{W}$ is more or equal to $2 m$, since it includes all the edges that leave $\mathcal{W}$ plus the edges from the outside. Taking into account that each non-terminal vertex has either one or two incoming edges, we conclude that the amount of the incoming edges could not be more than $2 m$ so it is $2 m$ exactly and every vertex from $\mathcal{W}$ has got exactly 2 incoming vertices. So we immediately get two results: If wandering vertices exist, then they comprise an isolated subgraph (i.e. it is impossible to get into there from any vertex outside of this subgraph); second, each wandering vertex has got exactly two predecessors, and hence each wandering vertex corresponds to the state in which each player has at least two cards.

Now pick up any wandering vertex and conduct the following 'back-tracking' procedure. If the vertex has two direct predecessors, consider the one in which 
the left player has less cards than in the current state (that is, the left player won the preceding play). Observe that this is always possible (see Fig. 5), as we can always backtrack through the play in which the left player was the winner. If we continue going back in this manner, we will finally reach the state in which the left player has only one card left, and hence has only one direct predecessor; this can not correspond to a wandering vertex. This means that a wandering vertex can be reached from a non-wandering, which contradicts our earlier finding (that wandering vertices constitute an isolated subgraph). This can only mean that the graph does not have any wandering vertices at all, and hence for each state there is a path to a final one.

Now we can use a well-established fact (see [3, Chap.3]) that if a graph corresponding to a finite Markov chain is absorbing, the mathematical expectation of the number of moves needed to reach the final state is finite, which is exactly what we expected to prove in the first place.

In particular, we prove that in the game with one suit the player having the highest card wins in a finite time. Zdes sleduet otmetit, chto eto utverzhdenie ne verno v Russkoi versii igri, v kotoroi edinstvennim otlichiem yavlyaetsya to, chto starhaya karta proigrivaet samoi mladshei karte. Konechno, i dlya etoi versii veren nash, resultat o konechnosti igri. !!!!!!!!!

We will not discuss the proof for the classical game of war in detail because the basic mathematical ideas are already contained in the proof of the model game (For details see [5, p.8]) We only note that the main idea in studying the real card game is the following obvious statement. If a subgraph of an oriented graph, which consists of all the vertices of the original graph, and might not include some of the edges, does not have any wandering vertices, then the original graph does not have them either.

We would like to point out that this card game was studied by other authors, however, they focused on other aspects of the game. In particular, Jacob HaqqMisra 2] uses numerical simulation (employing Monte-Carlo method) to find out how the advantage in the initial distribution of cards influences the outcome of the standard game; Ben-Naim and Krapivsky [1] discuss a stochastic model of the game. Very recently Michael Z. Spivey [4] has shown the possibility of cycles in rather general assumptions about the rules and the number of cards.

It is a curious observation that when the rules of the game allow the lowest valued card in the pack to win over the highest one, it is possible that with the same initial setting either of the players can win. An example of such situation is provided on Fig. 7 .

Finally let us note that for every $n$ the probability to keep playing after $n$ steps is always positive. It follows from the fact that the graph of the game has cycles 2 To show this, it is enough to pick any non-final state and transition to any of the ancestors. The existence of a cycle follows from the finiteness of the graph.

\footnotetext{
${ }^{2}$ We suppose that initial state is arbitrary
} 


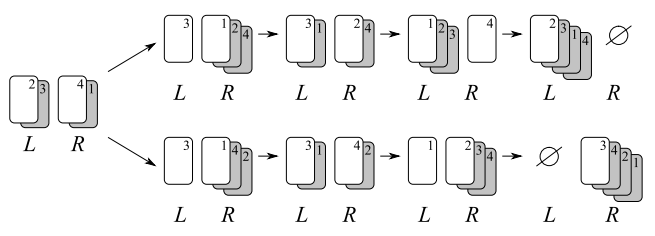

Figure 7: A game with two possible outcomes: $n=4$, the lowest valued card in the pack wins over the highest valued one.

Acknowledgments. The authors are grateful to the first author's son Grigory Lakshtanov (5 years old) for tireless practical experimentation searching for cycles with his father, and to the second author's partner Ian Robson for his help with correcting the authors' English.

\section{References}

[1] E. Ben-Naim, P.L. Krapivsky, Parity and ruin in a stochastic game, Eur. Phys. J. B 25, (2002) 239-243.

[2] Jacob Haqq-Misra, Predictability in the game of war, Scientific Creative Quarterly, 4, (2009)

[3] J.G.Kemeny, J.L.Snell, Finite Markov Chains, Springer-Verlag, 1976.

[4] Michael Z. Spivey, Cycles in War, Integers. (10), (2010) 747-764.

[5] E.Lakshtanov, V. Roshchina, Finiteness in the Card Game of War, arXiv:1007.1371[math.DS] (2010).

Evgeny Lakshtanov received his Ph.D. from the Moscow State University in December 2004. He currently does his posdoctoral research in the University of Aveiro, Portugal. On schitaet samim vazhnim resultatom svoei raboti sozdanie of simplified versions of serious mathematical models to make them understandable by children, preserving both aesthetic and intellectual value. The latter is in particularly measured by whether a given simplification allows setting a sufficient list of problems feasible for school students.

Center for Research and Development in Mathematics and Applications (CIDMA), Department of Mathematics, Aveiro University, Portugal.

lakshtanov@ua.pt

Vera Roshchina received her... When she is not doing mathematics, she enjoys singing, bicycling, and playing volleyball

Research Center in Mathematics and Applications (CIMA), University of Evora, Portugal. 


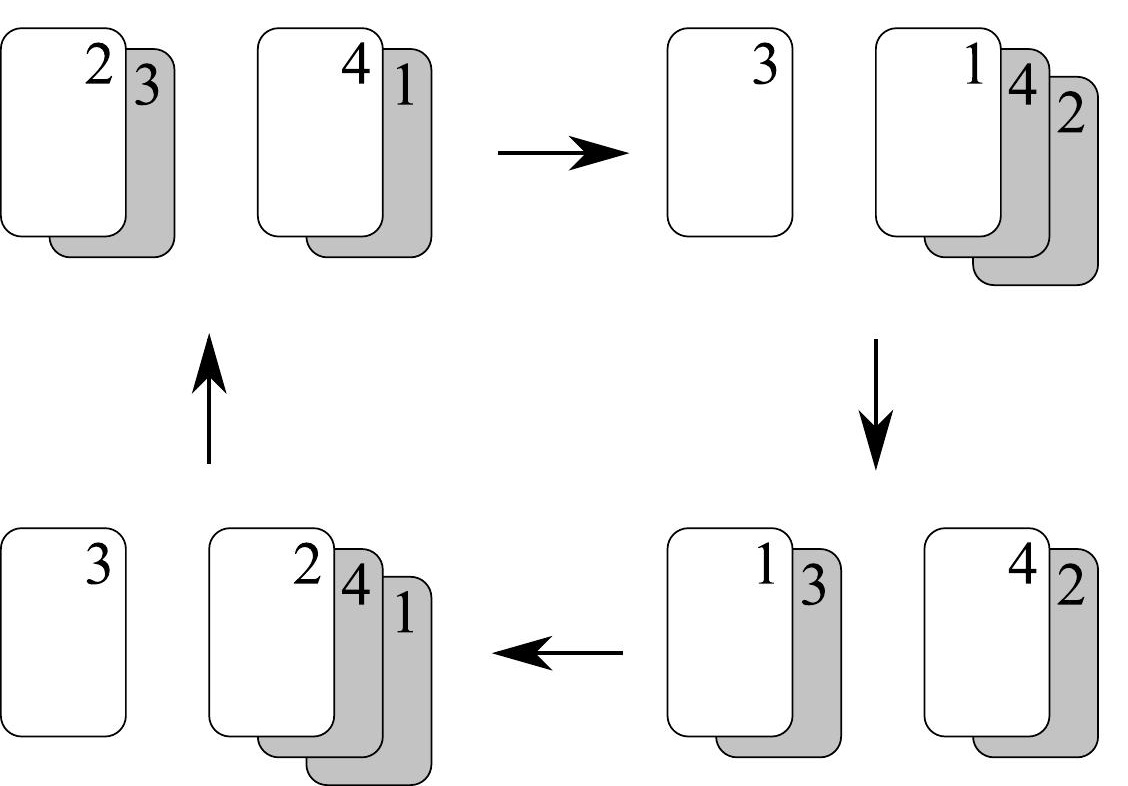

